\title{
RECENT CYBER SECURITY BREACHES AND PREVENTIVE MEASURES
}

\author{
N. Thirupathi Rao and Debnath Bhattacharyya \\ Department of Computer Science and Engineering, \\ Vignan's Institute of Information Technology $(A)$, \\ Visakhapatnam 530049, AP, India. \\ nakkathiru@gmail.com,debnathb@gmail.com
}

\begin{abstract}
A data breach event can be treated as a confirmed action or event happened such that some confidential or secret or protective data has been utilized or accessed by an unauthorized persons. The data that can be considered breaching of data like the credit card information, personal information for a person identification, health care data, patients data for a severe diseases, manufacturing devices which have huge demand in market and other software products related data. In the current article an attempt has been made to provide the reasons for breach of a data and the recent issues that were happened to be noted and also the preventive measures to be followed to protect the valuable data from these data breaches is also presented.
\end{abstract}

Keywords- Cyber security, data breach, personal information, medical information, credit and debit card information etc

\section{INTRODUCTION}

The growth of technology had increased a lot in recent years in various technological areas. Some of those areas like the software industry, manufacturing industry, banking sector and social marketing and social networking etc, $[1,2,3]$. As the growth of these areas is being increasing, in the similar fashion the other areas which were directly or indirectly related to such areas are also growing in a good manner. The technologies related to these areas are also growing in a rapid manner and the utilization of such networks and such websites for utilizing such features is also increasing a lot $[13,14,15]$. The number of people being utilizing such social networking websites and social marketing websites and other applications related to internet based working also have a scope of cheating and access the data that can be kept as secret in their personal accounts [4]. The data that can be posted in their personal websites or in personal accounts also can be prone of accessing such data and can be used for various other anti social events or for blackmailing such users for money is also a common issue becoming on daily basis [16, 17]. 


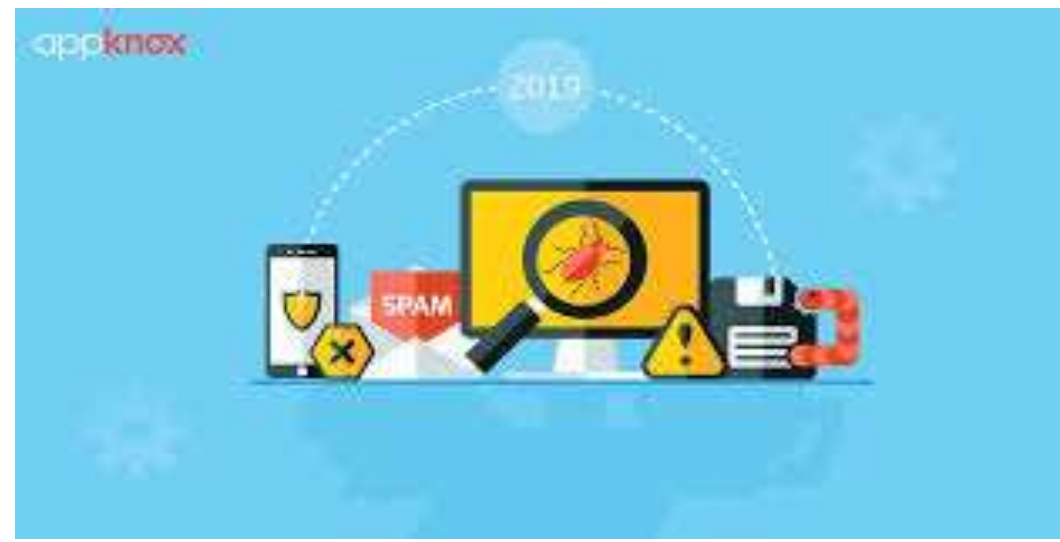

Fig. 1 Cyber attack models example [5]

The word data breach is becoming very serious issue on these days. The personal data of a person can be accessed or being watched by another person without having proper permissions or proper accesses and accessing such confidential data without the knowledge of the actual person. The data might be accessing without the proper login credentials or proper authorization and the data can be used for some other purposes. The data breaches can be happened in various cases with various scenarios. An attacker is being entering in tot the database of a software company and accessing all the data regarding the employees and the works that were being carried out there in such companies is a big data breach $[17,18]$. The other issue may be some unauthorized person is accessing the information about a patient $\mathrm{n}$ a hospital without the knowledge of the local doctors or the staff in the hospital. The other type of serious issues that might happen is the phishing emails through social engineering or social networking. Through these social networking and social engineering the people will meet or can chat and continue their friendships to next levels where they can share their personal login credentials of emails and other social network passwords. The attackers can then use their personal details and can login to their mail accounts and other social network accounts and can damage or stole the data or can be misused for some anti social events.

Some of the reasons for happening such events are the weak passwords for the accounts, missing patch files of the login credentials, mobile devices which were stolen and also the stolen computers or the inside hard disk drives [11, 12]. The criminals can use these sorts of data for breaching the people by stealing their data and committing anti social events with such stolen data [19]. The other type of chances for committing such breaches are like accessing through the third party organizations through which the actual big organizations data and other important files can be attacked and can be use for some other bad purposes [20]. The people who were committing such mistakes are considered as the hackers and cyber criminals. Some organizations intentionally keep their data available to the cyber criminals such that when they were being attempted to access such data, the persons or their location can be identified such that catching or arresting of such criminals might becomes sometimes more easy. 


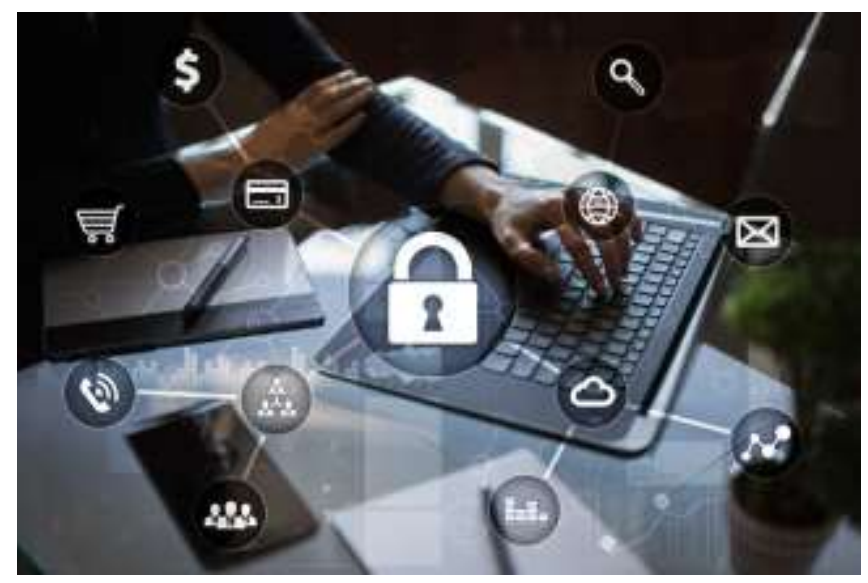

Fig. 2 Cyber security example model [4]

Several governments had derived various organizations such that they can guide and can handle the data or in some cases the sensitive data. Various governments had given strict and stringent rules for maintaining the data and various rules and regulations to maintain the data that can be avoided by the hackers. Some governments had designed a separate department to monitor and regulate the data breaches especially with the credit card related issues [5]. They had strictly given the direction such that who can have the data about the credit cards numbers, pins and other confidential data. For monitoring such events they had formed a separate section called the Payment Card Industry Data Security Standard. By using this section, the full operations related to credit card purchasing, payments, personal data, card data being handled and utilization was strictly processed and given rules to who can control such units.

Several governments had developed various departments and the common precautionary rules for protecting the confidential data about the credit card frauds, health information frauds, personal information frauds and other types of data breaches. The organizations who ever are working on such areas of functioning should follow these sorts of strict and stringent rules such that the privacy and the protection of the customers or the citizen's data will be protected.

\section{VARIOUS DATA BREACH INCIDENTS}

Some of the important and serious data breaches which were taken place recently are given in detail as follows,
1. Toyota's Second Data Breach
2. The Phishy Wipro Breach
3. Walmart Email Breach
4. The Citrix Breach
5. Blur Data Breach
6. JustDial Data Leak

\subsection{TOYOTA'S SECOND DATA BREACH}

Several giant companies in the worlds are also not exceptions for these sorts of data breaches. One of the giant manufacturing company the Toyota had announced officially in March 2019 that their customer's data was hacked and the data being stolen. They also estimated the number of people or the customers might had affected with this data breach was about 3 million customers. Also later they had announced that the credit card details of such customers were not present in such servers which were prone of attacks and their data was safe. Several days the engineers in the company had tries to assess the damage 
being caused and the amount of data being accessed by the hackers. At last they confirmed that the credit card information stored in the server was not been hacked and the server which had hacked was containing only the customers details not about the financial details.

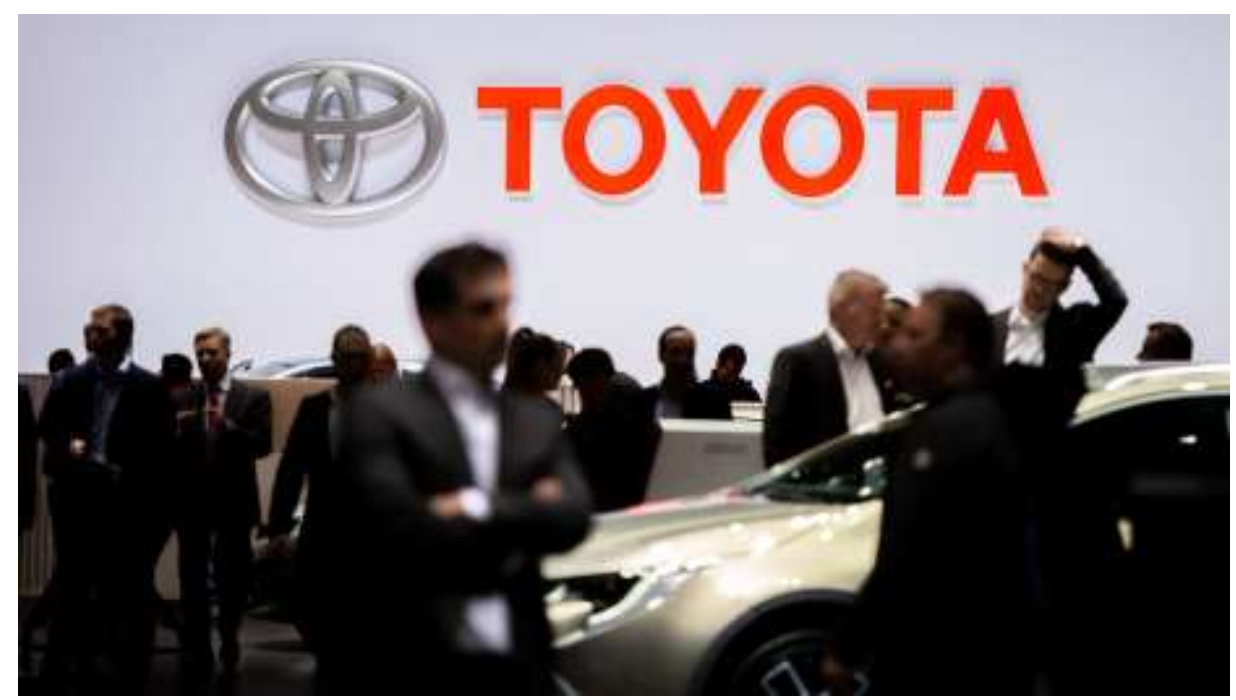

Fig. 3 Data Breach in Toyota Company example [6]

The same company had faced another cyber attack by the attackers in Australia in February 2019. The attack was identified when there is a similar type of press note was being placed in the internet for the customers like the Toyota Company want to place. As the news spread outside the company had analyzed the hacked data and the servers and confirmed that the personal data of the customers had not compromised and also they had informed to all the cyber security experts around the worlds to identify and warn the other source s of companies to prevent for their organizations for similar sorts of attacks might happen again. The lessons learned from such data breach in such companies was the Toyota had announced officially that whatever the communication is being coming to the customers from the name of the organization, the company requested the customers to confirm the same with the company executives or the customer care services of the company such that the fraud or wrong callers or information can be reduced.

\subsection{THE PHISHY WIPRO BREACH}

There are some Indian Giant software companies or firms who were suffering with these sorts of data breaches. One of such companies was the Wipro Ltd. The breach had happened with the customers of the company. Some of the machines in the company are being hacked by the intruders and the data being stolen and with such data, the hackers tried to hack and access the data of several other clients of the company such that financial sources of the clients can be accessed and can be hijacked. It is said that about a dozen computers of the company had been hacked and the important data about the customers of the company had been stolen. Later, the company had confirmed it and it promised the security and the confidentiality of the customers of their company would be maintained more securely and all the other security features and options to be provided for them will be provided on the immediate basis. The company also confirmed that it had hired some outside agencies also to identify the damage and also to support further for such sorts of events to be not happened in the near future. 


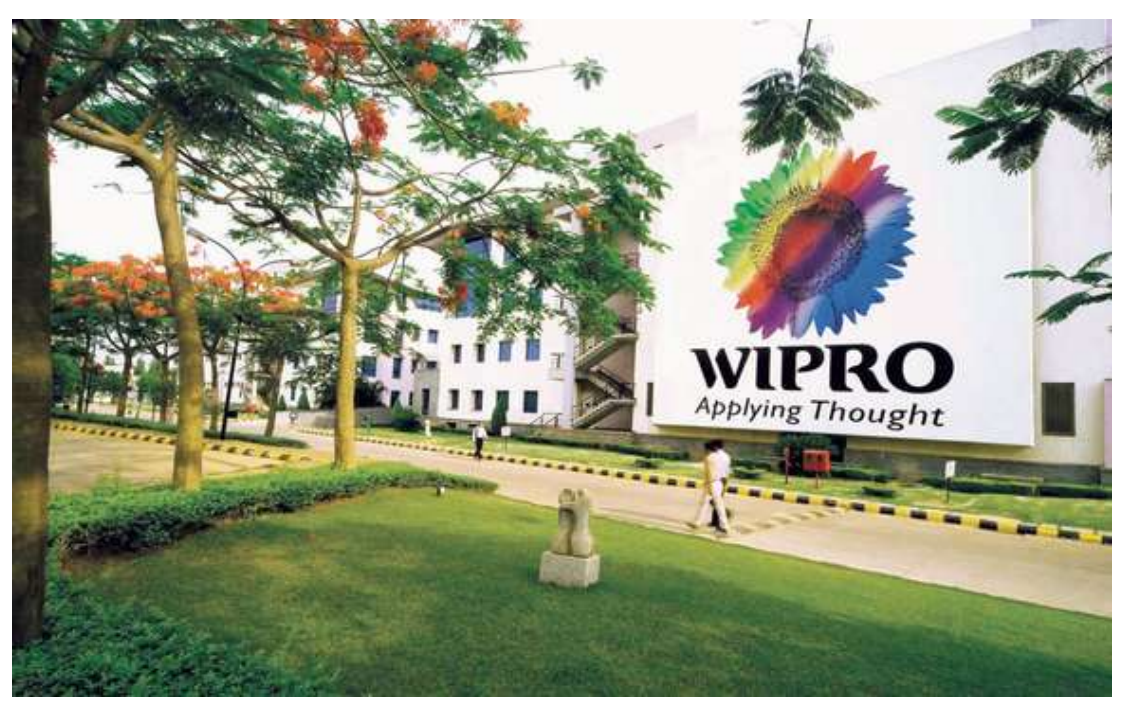

Fig. 4 Wipro Breach example [7]

In the later stages, the company had considered several preventive measures such that the similar sort's of events may not happen regularly. The company also confirmed that the event had happened with the wrong utilization of some employees and the phishing had happened for such systems. Very little number of machines was being attacked and the results can be estimated as very less damage to the customers and clients' data and their account details. The Wipro had confirmed and assured their clients that the similar vents may not happen again in the coming near future and the some strict and safe security measures will be taken such that to not to repeat the similar sort of events. The Wipro had also suggested several preventive measures to its customers and clients for dealing such sorts of Phishy calls and unauthorized calls and their data collections in the near future.

\subsection{Walmart Email Breach}

An email based data breach was observed in the world's famous giant organization Walmart. It is observed that in the last months of 2015 and the early months of 2016, some of the email addresses and the customer's data being hacked and being monitored by some suspicious attackers from outside the organization [8]. Later they had identified that the companies doubt was correct and the data of the customers being watched and hacked by the employees in the organization without the knowledge of the current Walmart organization. They had identified that the customers data and the emails of the customers are being hacked by the other people other than the company was observed when some one of the employee of the other organization. The Walmart had assigned a CompuCom company as their customer care service agency to contact customers for their satisfaction and service of the organization being offered. 


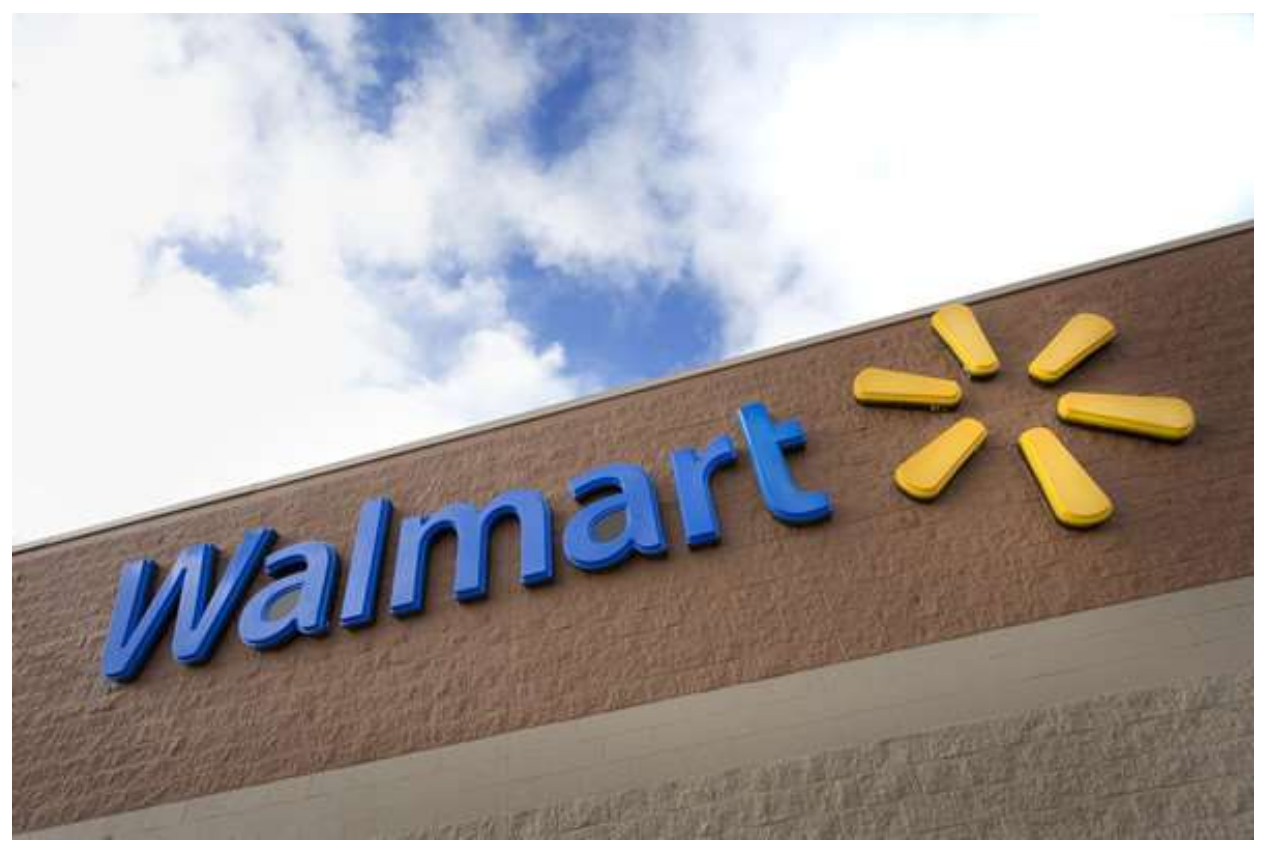

Fig. 5 Walmart Email Breach [8]

It is observed and it came to the notice of the outside world when one of the employees of CompuCom Company had a photo of the email details of the customers of the Walmart. The details were tapped and the similar set of data was being collected and the data was being misused. Hence, the organization had observed such events seriously and attempted to stop such events not to happen again in the near future. Later the Walmart organization had given the news that it had closed the contract with the CompuCom company and also given that it is going to procure some more software security tools such that to protect their customers data more confidentially and cannot be traced or misused by any other third party organizations.

\subsection{THE CITRIX BREACH}

The other serious data breach happened was the International Citrix Network where the data of the company like the emails, data storage, blue prints of the company models and other classified data of the company. The targeted company which committed this breach was the Iranian network hackers group. This incident had happened in the December 2018 and again the second time happened in the early months of 2019. The several federal agencies had identified the problems with these hackers and informed the organization for the threat and the data being hacked. In the later days the company had confirmed the same and data can be retrieved soon. 


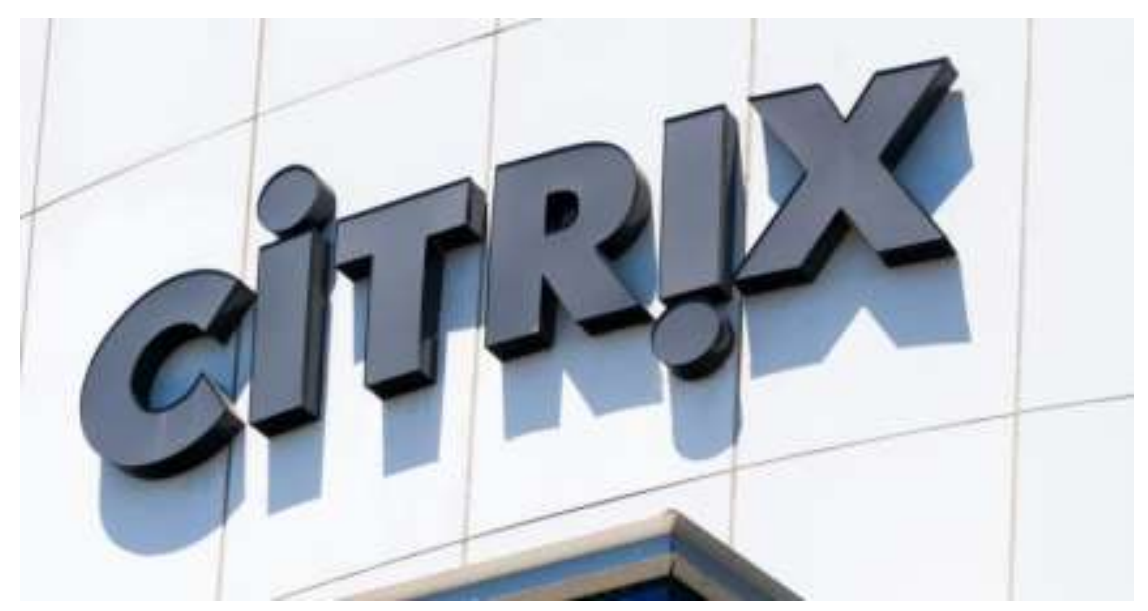

Fig. 6 The Citrix Organization [9]

The company later observed that around 6 Tera Bytes of data had been hacked by the hackers. An Iranian hacking group was behind this event and the same hacker group had targeted other agencies like the Oil and Gas companies around the world, technology companies around the world etc. The major way of getting access to these companies' websites and servers for accessing the data was by giving or sending multiple numbers of input requests at a time and get access to the servers and the data being stolen from such sources.

\subsection{BLUR DATA BREACH}

Another biggest data breach incident happened was the Blur Data Breach. The incident took place in the early months of 2019. The source for this incident was observed in the month of December 13, 2018. Around 2.5 million customers of the Blur organizations data had been breached [10]. The incident came in the live when a security expert sent a mail to the organization saying that they had observed the file in the network containing the user details like the email details, address of contact and other personal information of their customers. After thorough enquiry, the Abine company who was providing the login details security to the company had confirmed the same.

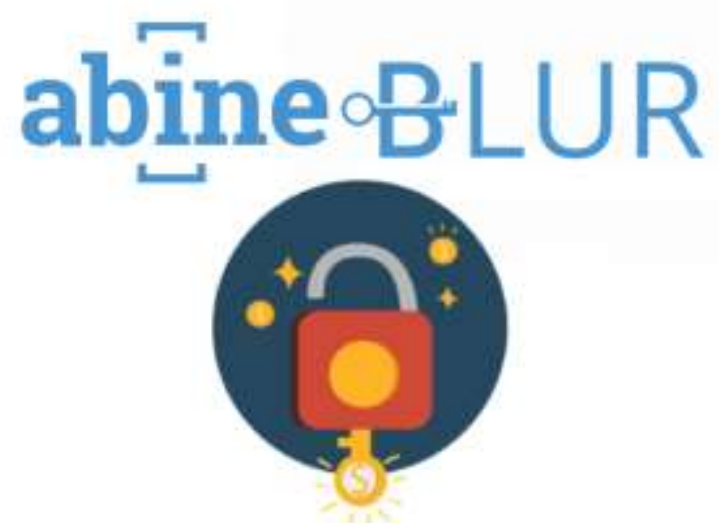

\section{ABINE'S BLUR PASSWORD MANAGER, LATEST VICTIM OF DATA BREACH}

Fig. 7 Abine Blur Data Breach Example [10] 
The company said that based on the data given by the security expert, it had conducted an internal audit and confirmed that the data breach had happened and requested all the customers to change their login credentials and other security details kept in their accounts. The data that was being hacked is containing data like the user name and passwords of the customers, personal details of the customers, password hints, ip addresses and the second ip addresses, Blur encrypted passwords etc. Later the Abine company had requested all its customers to change their passwords and always follow the two level security procedure to safe guard themselves for further breach of data again.

\subsection{JUSTDIAL DATA LEAK}

Another important data breach event happened in India with the customer service organization JustDial very recently [11]. Around 100 Million users data was being hacked and being made available publicly in the internet for various sources and applications. More than $80 \%$ of the customers who called for the customer care are breached and their data was lost to the hackers. Even if the person had used the application for one time or not used even for one time also, the customer's data being hacked. Later after thorough investigation, it was found that the data was being hacked form one of the old servers of the company which were not being using today and the entire data from such servers had breached.

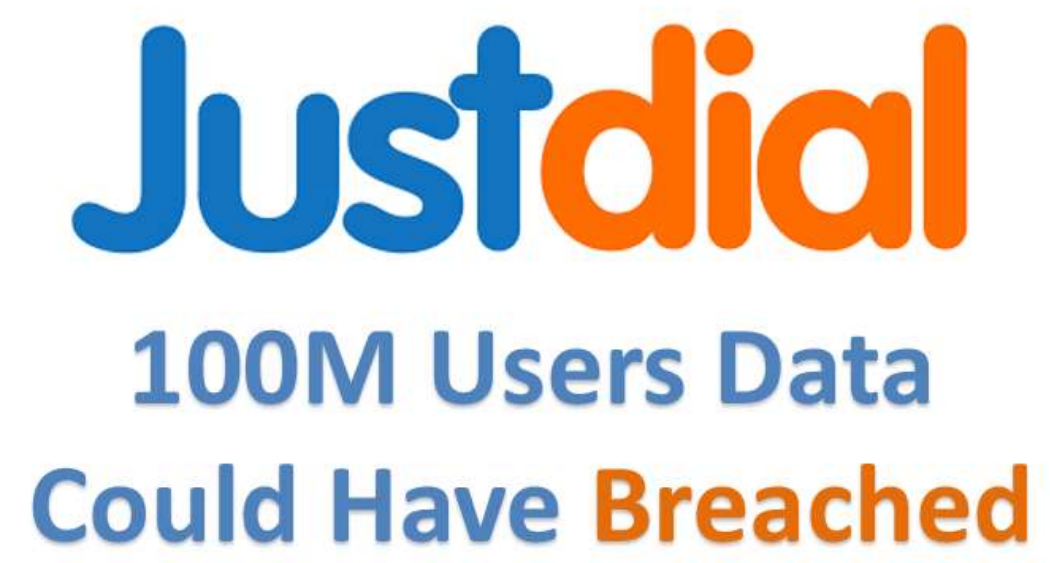

Fig. 8 JustDial Application Data Breach example [11]

However, the company had denied its data breach and said all its applications and these applications related servers are updated and up to date and there is no chance of having or happening such data breach. Also, it stated that very few customers from old servers are being active and almost all the customers are being using the new servers and there is no chance of happening such data breach. The company also confirmed that they had conducted some internal audit and enquiry such that this sorts of data breach had happened or not and then they confirmed that the data breach had not happened.

\section{STEPS TO BE FOLLOWED TO AVOID OR PREVENT FROM DATA BREACHES}

In order to reduce or prevent from such serious data breaches, there were several procedures or steps can be followed to escape or being protected from such data breaches. Some of those are given as follows, 


\section{a. Patching and updating software as soon as options are available}

It is always recommended for the users with mobile phones or computer systems to update their software's whenever there is an update for the existing software is available.

\section{b. Enforcing strong credentials and multi-factor authentication}

Whenever the users are setting or creating their accounts either in online accounts or in other website models, the users need to provide the strong passwords such that the breaking of such passwords will be almost difficult like the combinations of alphabets with both upper and lower cases, and also the combinations of alphabets and numerical numbers and with some special characteristics.

\section{c. Updating the software whenever a software being given by the manufacturer}

Whenever software update or a patch file was being given by the manufacturer, the employees or the users of such software's should immediately use the updates and the other files also be updated as per the requirements.

\section{CONCLUSIONS}

In the current article, an attempt has been made to provide the details regarding the data breaches that were happened in recent years. The problems that were faced by those companies also discussed in detail. The preventive measures to be taken such that to avoid these sorts of attacks also can be given in the current article. Several data breaches that were happened in and around the world are discussed in detail.

\section{REFERENCES}

[1] https://www.cshub.com/attacks/articles/top-5-cyber-security-breaches-of-2019-so-far [Last Accessed on 10-07-2019]

[2] https://www.csoonline.com/article/2130877/the-biggest-data-breaches-of-the-21st-century.html [Last Accessed on 11-07-2019]

[3] https://searchsecurity.techtarget.com/definition/data-breach [Last accessed on 15-07-2019]

[4] https://www.openaccessgovernment.org/inevitable-cyber-security-breaches/58185/ [Last accessed on 10-08-2019]

[5] https://www.appknox.com/blog/top-cybersecurity-data-breaches-2019[Last accessed on 10-08-2019]

[6] https://www.axios.com/toyota-second-data-breach-bf45c49e-d1dc-48f7-932e-3fda0b31c8ee.html [Last Accessed on 10-08-2019]

[7] https://www.computing.co.uk/ctg/news/3074239/wipro-investigates-security-breach-believed-to-beperpetrated-by-state-sponsored-attacker [Last Accessed on 01-08-2019]

[8] https://www.cshub.com/attacks/articles/incident-of-the-week-investigation-of-walmart-email-breach [Last Accessed on 10-08-2019]

[9] https://nakedsecurity.sophos.com/2019/03/12/citrix-admits-attackers-breached-its-network-what-weknow/ [Last Accessed on 10-08-2019]

[10] https://hackercombat.com/abines-blur-password-manager-latest-victim-of-data-breach/ [Last accessed on 01-08-2019]

[11] https://hackersonlineclub.com/justdial-database-unprotected/[Last accessed on 10-07-2019]

[12] Wonjin Jung, "The Effects of the Perceived Accessibility and Cyber Security of Mobile Banking Applications on User Loyalty to the Applications", International Journal of Mobile Device Engineering. Vol. 2. No. 1. Mar. 2018.GVPress. pp:7-12.

[13] Jin-Hwan Jung, Seong-Pyo Kim, and Jung-Ho Eom, "Offensive Cyber Security Strategy according to the Evolution of Cyber Attack Technology", International Journal of Reliable Information and Assurance. Vol. 6. No. 2. Dec. 2018.GVPress. pp:1-6.

[14] Jin-young Song and Dea-woo Park, "Preemptive Cyber Response Strategy and IoT Forensic Evidence", International Journal of Advanced Science and Technology, SERSC Australia, Vol. 117, August 2018, pp. 129-138.

[15] Sabah Mohammed and Jinan Fiaidhi, "Cyber Physical Systems: A New Frontier of Artificial Intelligence: Summary Paper", International Journal of Control and Automation, NADIA, Vol. 12, No. 1, January 2019, Pp. 23-30. 
[16] Jung ho Eom, "Modeling of Cyber- attack Intentions Analysis Reflecting Domestic/International Situations", International Journal of Grid and Distributed Computing, SERSC Australia, Vol.11, No.1, January 2018, Pp. 13-26.

[17] Shailendra Singh and Sanjay Silakari, "Cyber Attack Detection System based on Improved Support Vector Machine", International Journal of Security and Its Applications, SERSC Australia, Vol.9, No.9, September 2015, Pp. 371-387.

[18] Yuyu Yin, Fangzheng Yu, Yueshen Xu, Lifeng Yu, Jinglong Mu, "Network Location-Aware Service Recommendation with Random Walk in Cyber-Physical Systems", Sensors, 2017 Vol.17, No.9, Pp. 2059-2064.

[19] Yu, Jun; Rui, Yong; Tang, Yuan Yan; Tao, Dacheng., "High-Order Distance-Based Multiview Stochastic Learning in Image Classification", IEEE Transactions on Cybernetics, 2014, Vol.44, No.12, Pp.2431-2442.

[20] Yu, Jun; Tao, Dacheng; Wang, Meng; Rui, Yong., "Learning to Rank Using User Clicks and Visual Features for Image Retrieval”, IEEE Transactions on Cybernetics, 2015, Vol.45, No.4, Vol.767-779. 